\title{
Article \\ Solvability for a Class of Integro-Differential Inclusions Subject to Impulses on the Half-Line
}

\author{
Paola Rubbioni (iD
}

Citation: Rubbioni, P. Solvability for a Class of Integro-Differential Inclusions Subject to Impulses on the Half-Line. Mathematics 2022, 10, 224. https://doi.org/10.3390/ math10020224

Academic Editors: Anca Croitoru, Radko Mesiar, Anna Rita Sambucini and Bianca Satco

Received: 10 December 2021

Accepted: 10 January 2022

Published: 12 January 2022

Publisher's Note: MDPI stays neutral with regard to jurisdictional claims in published maps and institutional affiliations.

Copyright: (C) 2022 by the author. Licensee MDPI, Basel, Switzerland. This article is an open access article distributed under the terms and conditions of the Creative Commons Attribution (CC BY) license (https:// creativecommons.org/licenses/by/ $4.0 /)$.
Department of Mathematics and Computer Science, University of Perugia, 06123 Perugia, Italy; paola.rubbioni@unipg.it

\begin{abstract}
In this paper, we study a semilinear integro-differential inclusion in Banach spaces, under the action of infinitely many impulses. We provide the existence of mild solutions on a half-line by means of the so-called extension-with-memory technique, which consists of breaking down the problem in an iterate sequence of non-impulsive Cauchy problems, each of them originated by a solution of the previous one. The key that allows us to employ this method is the definition of suitable auxiliary set-valued functions that imitate the original set-valued nonlinearity at any step of the problem's iteration. As an example of application, we deduce the controllability of a population dynamics process with distributed delay and impulses. That is, we ensure the existence of a pair trajectory-control, meaning a possible evolution of a population and of a feedback control for a system that undergoes sudden changes caused by external forces and depends on its past with fading memory.
\end{abstract}

Keywords: semilinear differential inclusions; impulsive problems; feedback controls; distributed delay; population dynamics

MSC: Primary: 34G20; 34G25; 34A37; Secondary: 92D25; 93B52

\section{Introduction}

In this paper, we study the existence of solutions to the semilinear integro-differential inclusion

$$
y^{\prime}(t) \in A(t) y(t)+F\left(t, y(t), \int_{t_{0}}^{t} k(t, s) y(s) d s\right), t \geq t_{0}
$$

with the initial condition $y\left(t_{0}\right)=\mathbf{v} \in E$, where $E$ is a real Banach space. Here, $\{A(t)\}_{t \geq 0}$ is a family of linear operators acting on the Banach space, $F:\left[t_{0},+\infty[\times E \times E \multimap E\right.$ is a given set-valued map, and $k$ is a nonnegative real function.

Currently, many researchers around the world are investigating the semilinear integrodifferential equations or inclusions, as witnessed, for example, by the recent articles [1-8]. One of the main reasons for this research is that these equations are well suited to serve as a model for real phenomena such as heat transfer or the spread of epidemics or population dynamics, in which it is significant to take into account the spatial diffusion of the phenomenon or the past of the phenomenon itself (e.g., $[9,10])$.

The need to introduce delays in models describing real phenomena has appeared clear since the beginning of the last century, due to the fact that some of the processes involved in the dynamics may depend on the past status of the population. Think, for example, of the study of a phenomenon in which only individuals of childbearing age are to be considered. Clearly, in this case, the time between birth and the moment when the individual is involved in the reproductive process is not irrelevant, leading to a non-negligible maturation delay influencing the evolution of the population over time. Among all the pioneers' works on delay equations, we wish to recall that of Volterra, from which a whole class of differential equations will take his name (see [11] for a historical review). In the decades following the 1950s, the delay equations have been studied either in the case of concentrated delay, 
distributed delay, or functional delay. We refer to the papers $[12,13]$ for the first studies concerning semilinear differential equations with delay, to the monographs [14-16] for a more in depth treatment on delay differential equations, and to [17] for the use of delays in population models.

Along this line, we will apply the results of the investigation on the semilinear integrodifferential inclusion (1) to the study of a population dynamics model described by the parametric integro-differential equation involving a distributed delay

$$
\frac{\partial u}{\partial t}(t, x)=-b(t, x) u(t, x)+g\left(t, u(t, x), \int_{t_{0}}^{t} \frac{e^{-(t-s) / T}}{T} u(s, x) d s\right)+\omega(t, x),
$$

subject to feedback controls given by

$$
\omega(t, \cdot) \in W(u(t, \cdot)) \text {. }
$$

Here, $u, b, \omega:\left[t_{0},+\infty\left[\times[0,1] \rightarrow \mathbb{R}\right.\right.$ and $g:\left[t_{0},+\infty[\times \mathbb{R} \times \mathbb{R} \rightarrow \mathbb{R}\right.$ are given functions, while $W: L^{2}([0,1]) \multimap L^{2}([0,1])$ is a multimap.

The real value $u(t, x)$ represents the population density at time $t$ and place $x$, the removal coefficient $-b(t, x)$ the death rate and displacement of the population, and the nonlinearity $g:\left[t_{0},+\infty[\times \mathbb{R} \times \mathbb{R} \rightarrow \mathbb{R}\right.$ the population development law.

The function $g$ includes a Volterra integral, and this is what formalizes the distributed delay in the model, providing a spanning effect by means of the memory kernel

$$
k(t, s)=\frac{e^{-(t-s) / T}}{T} .
$$

This kernel is given by the exponential distribution of probability $\mathcal{K}(\tau)=\frac{e^{-\tau / T}}{T}$. Since $\mathcal{K}$ is decreasing, the two-variable function $k$ assigns a greater weight to the most recent events, increasingly fading the influence of those further away in time. Note that this happens in a maximum range indicated by $T$. In fact, the positive number $T$ provides the width of the action of the kernel: the larger $T$ is, the more the system's memory is extended to past events affecting its present state. Thus, we can say that the value $T$ shows the range of significance of the delay. We point out that, inasmuch as the process is set on the whole half-line, the number $T$ can be chosen arbitrarily large. In other words, the relevance of the delay on the status of the solution trajectory can be chosen arbitrarily, thus making the model particularly versatile.

The set-valued function $W$ provides the sets where the feedback controls can be taken. Feedback controls often appear in models from the life Sciences, especially in systems biology. For a detailed description of the topic see [18].

Further, we consider the presence of infinitely many impulses on the system. These are represented by given functions acting in correspondence to times $t_{m}$, where $\left\{t_{m}\right\}_{m \geq 1}$ is an increasing diverging sequence of positive numbers, and leading to jumps on the solutions' functions according to relations for all $m \geq 1$

$$
\lim _{h \rightarrow 0^{+}} y\left(t_{m}+h\right)=y\left(t_{m}\right)+I_{m}\left(y\left(t_{m}\right)\right),
$$

or

$$
\lim _{h \rightarrow 0^{+}} u\left(t_{m}+h, x\right)=u\left(t_{m}, x\right)+\mathcal{I}_{m}\left(u\left(t_{m}, x\right)\right),
$$

in case we deal with the general integro-differential inclusion (1) or the model's parametric differential Equation (2), respectively.

Problems involving instantaneous impulses have been extensively studied in the literature and are still a topic of considerable interest, as can be seen in recent articles [19-25]. For a first approach to the subject, we refer to the now classic monographs $[26,27]$. The reason lies in that impulse functions are needed in the modeling 
of a wide range of real phenomena whenever an external factor that extends for a very short period of time - to the point of being considered instantaneous - intervenes to disturb the system, causing sudden changes in the evolution of the trajectories that describe the evolutionary dynamics of the process. For example, but not limited to them, impulse functions can represent the administration of antibiotics on a bacterial population in the treatment of a disease, or abrupt changes of prices in economics, or the use of pesticides in pre-established times to keep a pest in a crop below a certain threshold (in biology, these functions are called "regulation functions").

This paper is organized as follows:

The most important notions necessary to place the topics covered in the manuscript are shortly collected in Section 2.

Then, in Section 3, the Cauchy problem driven by the semilinear integro-differential inclusion (1) is formally stated, and the existence of mild solutions is provided, both in and out of the presence of impulses. We achieve our main existence result by means of an "extension-with-memory" process, which generates an impulsive mild solution starting from the mild solutions of an ordered iterative sequence of non-impulsive Cauchy problems. As far as we know, this method was first used in [28] and in [29] without memory and with functional delay, respectively, but on a compact interval in both cases. More recently, it has been firstly used on the half-line in [25] to provide the existence of mild solutions of an impulsive Cauchy problem driven by the semilinear differential equation with functional delay

$$
y^{\prime}(t)=A(t) y(t)+f\left(t, y(t), y_{t}\right), t \geq t_{0},
$$

where $y_{t}(\theta):=y(t+\theta), \theta \in[-\tau, 0], t \in\left[t_{0},+\infty[\right.$. Contrary to what one might think at first glance, the analogous problem governed by the semilinear integro-differential equation

$$
y^{\prime}(t)=A(t) y(t)+f\left(t, y(t), \int_{t_{0}}^{t} k(t, s) y(s) d s\right), t \geq t_{0}
$$

was still open, even on a compact interval. Indeed, the different nature between function $t \mapsto y_{t}$ and function $t \mapsto \int_{t_{0}}^{t} k(t, s) y(s) d s$ does not allow the same demonstration arguments to be used. In the present work, we provide an answer to this open problem, even in the multivalued case. The key of our procedure is given by the introduction of suitable auxiliary set-valued functions, which "imitate", satisfying its own properties (cf. next Lemma 1), the original set-valued nonlinearity $F$ at any step of the problems' iteration. Furthermore, we would like to point out that the extension-with-memory method enables the existence of solutions with no hypothesis on the impulse functions, unlike other approaches adopted in the literature, for which those functions are supposed to be at least continuous.

In Section 4, we consider the system governed by the parametric integro-differential equation with distributed delay (2) under the action of feedback controls and impulses. We solve the feedback control problem by rewriting the model as an impulsive Cauchy problem driven by a semilinear integro-differential inclusion in the space $E=L^{2}([0,1])$. In this way, we can apply the general result obtained in Section 3 and lead to the existence of a pair, trajectory-control, providing the controllability of the population dynamics process.

\section{Essential Preliminary Notions}

We recall some basic notations and definitions.

Let $X$ and $Y$ be two topological spaces. A set-valued function (or "multivalued map", or, shortly, "multimap") $\mathcal{F}: X \multimap Y$ is upper semicontinuous at $x_{0} \in X$ if, for every open $V \subset Y$ such that $\mathcal{F}\left(x_{0}\right) \subset V$, there exists a neighborhood $U$ of $x_{0}$ such that $\mathcal{F}(x) \subset V$ for every $x \in U$. A multimap $\mathcal{F}$ is upper semicontinuous if it is upper semicontinuous at every $x_{0} \in X$.

Let $E$ be a real Banach space endowed with the norm $\|\cdot\|$. By the symbol $C(J, E)$, we denote the space of $E$-valued continuous functions on a closed, bounded interval $J \subset \mathbb{R}$, 
while by $L^{p}(J, E)$ we denote the space of all functions $v: J \rightarrow E$ such that their $p$-power is Bochner integrable endowed with the norm $\|v\|_{L^{p}(J, E)}=\left[\int_{J}\|v(z)\|^{p} d z\right]^{\frac{1}{p}}$ (shortly, $L^{p}(J)$ and $\|v\|_{L^{p}}$, respectively, if $\left.E=\mathbb{R}\right), p \geq 1$. Moreover, for any $a \in \mathbb{R}$, by the symbol $L_{l o c}^{1}\left(\left[a,+\infty[, E)\right.\right.$, we mean the space of all functions $v:\left[a,+\infty\left[\rightarrow E\right.\right.$ such that $v \in L^{1}(J, E)$ for every compact $J \subset\left[a,+\infty\left[\right.\right.$ (shortly, $L_{l o c}^{1}([a,+\infty[)$ if $E=\mathbb{R})$. Then, throughout the paper for a given function $y:[a,+\infty[\rightarrow E$ and a fixed $t \in[a,+\infty[$, we will use the symbol

$$
y\left(t^{+}\right):=\lim _{h \rightarrow 0^{+}} y(t+h),
$$

whenever the limit exists.

A family $\{T(t, s)\}_{t \geq s \geq 0}$ of bounded linear operators on $E$ is said to be a (strongly continuous) evolution system on the half-line (see, e.g., [30]) if

(T1) $T(s, s)=I, \quad T(t, r) T(r, s)=T(t, s)$ for $t \geq s \geq 0$; and

(T2) for every $x \in E$, the map $\xi_{x}:(t, s) \mapsto T(t, s) x$ is continuous.

Further, a family of linear operators $\{A(t)\}_{t \geq 0}$ generates an evolution system on the half-line $\{T(t, s)\}_{t \geq s \geq 0}$ (see, e.g., [31]) if

$$
\frac{\partial T(t, s)}{\partial t}=A(t) T(t, s) \quad \text { and } \quad \frac{\partial T(t, s)}{\partial s}=-T(t, s) A(s), \quad t \geq s \geq 0 .
$$

We conclude this section recalling that the Hausdorff measure of noncompactness in $E$ is the function $\chi$ on the family of nonempty subsets of $E$ taking nonnegative real values defined by

$$
\chi(\Omega)=\inf \{\varepsilon>0: \Omega \text { has a finite } \varepsilon \text {-net }\}, \text { for all bounded } \Omega \subset E .
$$

The symbol $\chi_{L^{2}}$ will denote the Hausdorff MNC in $E=L^{2}([0,1])$. For the properties of the Hausdorff measure of noncompactness, we refer to [32].

\section{Existence of Impulsive Mild Solutions on the Half-Line}

Let $E$ be a real Banach space, and $\left\{t_{m}\right\}_{m \in \mathbb{N}}$ a set of fixed real numbers such that $0 \leq t_{0}<t_{1}<t_{2}<\ldots$ and $\lim _{m \rightarrow \infty} t_{m}=+\infty$. By the symbol $\mathcal{P C}\left(\left[t_{0},+\infty[, E)\right.\right.$, we denote the set of functions

$$
\mathcal{P C}\left(\left[t_{0},+\infty[, E):=\left\{\begin{array}{ll}
y:\left[t_{0},+\infty[\rightarrow E:\right. & y_{\left.\mid] t_{m-1}, t_{m}\right]} \text { is continuous, for all } m \in \mathbb{N}^{+} ; \\
& \exists \lim _{h \rightarrow 0^{+}} y\left(t_{m}+h\right) \equiv y\left(t_{m}^{+}\right) \in E, \text { for all } m \in \mathbb{N} .
\end{array}\right\}\right.\right.
$$

Let $\mathbf{v} \in E$ be fixed, and consider the corresponding initial value problem driven by a semilinear integro-differential inclusion subject to impulses $I_{m}: E \rightarrow E, m \in \mathbb{N}^{+}$at the given times $\left\{t_{m}\right\}_{m \in \mathbb{N}^{+}}$

$$
(P)\left\{\begin{array}{l}
y^{\prime}(t) \in A(t) y(t)+F\left(t, y(t), \int_{t_{0}}^{t} k(t, s) y(s) d s\right), t \geq t_{0}, t \neq t_{m}, m \in \mathbb{N}^{+}, \\
y\left(t_{0}\right)=\mathbf{v} \\
y\left(t_{m}^{+}\right)=y\left(t_{m}\right)+I_{m}\left(y\left(t_{m}\right)\right), m \in \mathbb{N}^{+} .
\end{array}\right.
$$

In this Section we suppose that:

(A) $\mathcal{A}:=\{A(t)\}_{t \geq 0}$ is a family of linear operators, $A(t): D(A) \subset E \rightarrow E, D(A)$ dense subset of $E$ not depending on $t$, generating an evolution system on the half-line $\{T(t, s)\}_{t \geq s \geq 0} ;$

(F) $F:\left[t_{0},+\infty[\times E \times E \multimap E\right.$ is a multimap satisfying the properties:

(F0) $F$ takes compact and convex values; 
(F1) for every $v, w \in E$, the multimap $F(\cdot, v, w)$ admits a strongly measurable selection;

(F2) for a.e. $t \in\left[t_{0},+\infty[\right.$, the multimap $F(t, \cdot, \cdot)$ is upper semicontinuous;

(F3) there exists a nonnegative function $\alpha \in L_{l o c}^{1}\left(\left[t_{0},+\infty[)\right.\right.$ such that, for a.e. $t \geq t_{0}$ and all $v, w \in E$,

$$
\|F(t, v, w)\| \leq \alpha(t)(1+\|v\|+\|w\|),
$$

where $\|F(t, v, w)\|:=\sup \{\|w\|: w \in F(t, v, w)\} ;$ and

(F4) there exists a nonnegative function $h \in L_{l o c}^{1}\left(\left[t_{0},+\infty[)\right.\right.$ such that

$$
\chi\left(F\left(t, \Omega_{1}, \Omega_{2}\right)\right) \leq h(t)\left[\chi\left(\Omega_{1}\right)+\chi\left(\Omega_{2}\right)\right],
$$

for a.e. $t \geq t_{0}$ and every bounded $\Omega_{1}, \Omega_{2} \subset E$;

(k) $k: \Delta_{\infty} \rightarrow \mathbb{R}^{+}, \Delta_{\infty}=\left\{(t, s) \in \mathbb{R}^{2}: t \geq s \geq t_{0}\right\}$, is a continuous function

We study the existence of mild solutions to $(P)$, according to the following definition:

Definition 1. A function $y \in \mathcal{P C}\left(\left[t_{0},+\infty[, E)\right.\right.$ is said to be a mild solution to $(P)$ if

$$
y(t)=T\left(t, t_{0}\right) \mathbf{v}+\sum_{t_{0}<t_{m}<t} T\left(t, t_{m}\right) I_{m}\left(y\left(t_{m}\right)\right)+\int_{t_{0}}^{t} T(t, s) f(s) d s, t \geq t_{0},
$$

where $f:\left[t_{0},+\infty\left[\rightarrow E\right.\right.$ is a $L_{\text {loc }}^{1}$-function on $\left[t_{0},+\infty[\right.$ such that

$$
f(s) \in F\left(s, y(s), \int_{t_{0}}^{s} k(s, \tau) y(\tau) d \tau\right) \text { for a.e. } s \geq t_{0}
$$

with the agreement that $\sum_{t_{0}<t_{m}<t} T\left(t, t_{m}\right) I_{m}\left(y\left(t_{m}\right)\right)=0$ if $t \in\left[t_{0}, t_{1}\right]$.

Note that every mild solution also satisfies the conditions.

$$
\begin{aligned}
& y\left(t_{0}\right)=\mathbf{v} ; \\
& y\left(t_{m}^{+}\right)=y\left(t_{m}\right)+I_{m}\left(y\left(t_{m}\right)\right), m \in \mathbb{N}^{+} .
\end{aligned}
$$

Before stating the main theorem of this section, we provide a preliminary result. It will be a strategic and decisive tool in applications. Indeed, thanks to the property stated by Lemma 1, we can apply the extension-with-memory technique (later shown) to integro-differential equations or inclusions having a two-variables kernel inside the Volterra integral, which is new in the literature, as far as we know. As a consequence, our existence theorem will be allowed to operate in a much wider class of models than is possible with the current results, to our knowledge.

Lemma 1. Let $E$ be a real Banach space, and $\left\{t_{m}\right\}_{m \in \mathbb{N}}$ a sequence of real numbers such that $0 \leq t_{0}<t_{1}<t_{2}<\ldots$ and $\lim _{m \rightarrow+\infty} t_{m}=+\infty$.

Assume that $F:\left[t_{0},+\infty\left[\times E \times E \multimap E\right.\right.$ and $k: \Delta_{\infty} \rightarrow \mathbb{R}^{+}$, respectively, satisfy $(F)$ and $(k)$.

Then, for every $m \in \mathbb{N}^{+}$and every set of functions $\left\{y_{i} \in C\left(\left[t_{i}, t_{i+1}\right], E\right): i=0, \ldots, m-1\right\}$, the multimap $F_{m}:\left[t_{m}, t_{m+1}\right] \times E \times E \multimap E$ defined by

$$
F_{m}(t, v, w):=F\left(t, v, w+\sum_{i=0}^{m-1} \int_{t_{i}}^{t_{i+1}} k(t, \tau) y_{i}(\tau) d \tau\right), t \in\left[t_{m}, t_{m+1}\right], v, w \in E
$$

satisfies $(F)$ in its $\left[t_{m}, t_{m+1}\right]$-restricted version.

Proof. Let $m>0$ and $\left\{y_{i} \in C\left(\left[t_{i}, t_{i+1}\right], E\right): i=0, \ldots, m-1\right\}$ be fixed. For the sake of simplicity, we denote the properties on $\left[t_{m}, t_{m+1}\right]$ by $(\mathrm{F} 0), \ldots,(\mathrm{F} 4)$, as the corresponding on $\left[t_{0},+\infty[\right.$. Clearly, property (F0) trivially holds. 
Then, let us fix $v, w \in E$ and consider the multimap on $\left[t_{m}, t_{m+1}\right]$ (see (6))

$$
F_{m}(\cdot, v, w)=F\left(\cdot, v, w+\sum_{i=0}^{m-1} \int_{t_{i}}^{t_{i+1}} k(\cdot, \tau) y_{i}(\tau) d \tau\right) .
$$

We notice that it can be seen as the Nemytskii superposition operator $N:\left[t_{m}, t_{m+1}\right] \rightarrow$ $E$ of the function $F_{v}:\left[t_{m}, t_{m+1}\right] \times E \multimap E$,

$$
F_{v}(t, \eta):=F(t, v, \eta), t \in\left[t_{m}, t_{m+1}\right], \eta \in E
$$

by the function $q_{w}:\left[t_{m}, t_{m+1}\right] \rightarrow E$,

$$
q_{w}(t):=w+\sum_{i=0}^{m-1} \int_{t_{i}}^{t_{i+1}} k(t, \tau) y_{i}(\tau) d \tau, t \in\left[t_{m}, t_{m+1}\right] .
$$

In fact, we get

$$
N(t):=F_{v}\left(t, q_{w}(t)\right)=F\left(t, v, q_{w}(t)\right)=F_{m}(t, v, w), t \in\left[t_{m}, t_{m+1}\right]
$$

Obviously, by (F0) of $F$ we have that $F_{v}$ takes compact values, by $(\mathrm{F} 1)$ that $F_{v}(\cdot, \eta)$ has a strongly measurable selector for every $\eta \in E$, and by $(\mathrm{F} 2)$ that $F_{v}(t, \cdot)$ is upper semicontinuous for a.e. $t \in\left[t_{m}, t_{m+1}\right]$.

Moreover, $q_{w}$ is strongly measurable; indeed, the functions $f_{i}:\left[t_{m}, t_{m+1}\right] \times\left[t_{i}, t_{i+1}\right] \rightarrow$ $E, i=0, \ldots, m-1$, defined by

$$
f_{i}(t, \tau)=k(t, \tau) y_{i}(\tau),(t, \tau) \in\left[t_{m}, t_{m+1}\right] \times\left[t_{i}, t_{i+1}\right]
$$

are continuous on $\left[t_{m}, t_{m+1}\right] \times\left[t_{i}, t_{i+1}\right]$ as product of continuous functions (cf. (k)). Thus, $q_{w}$ is in turn continuous on $\left[t_{m}, t_{m+1}\right]$ and hence strongly measurable.

It is therefore possible to apply Theorem 1.3.5 of [33] and claim that $N$ has a strongly measurable selector. Thus $F_{m}$ satisfies (F1).

Now, let us fix $t \in\left[t_{m}, t_{m+1}\right]$ such that $F(t, \cdot, \cdot)$ is upper semicontinuous, and consider the multimap on $E \times E$ (see (6) again)

$$
F_{m}(t, \cdot, \cdot)=F\left(t, \cdot, \cdot+\sum_{i=0}^{m-1} \int_{t_{i}}^{t_{i+1}} k(t, \tau) y_{i}(\tau) d \tau\right) .
$$

Since the vector

$$
w_{0}:=\sum_{i=0}^{m-1} \int_{t_{i}}^{t_{i+1}} k(t, \tau) y_{i}(\tau) d \tau
$$

is a fixed element in $E$, the map $w \mapsto w+w_{0}$ is just a translation function, thus $F_{m}(t, \cdot, \cdot)$ is the composition of a continuous single-valued function and an upper semicontinuous multimap. Thus, it is upper semicontinuous as well. Hence, property (F2) is satisfied by $F_{m}$.

In order to prove that $F_{m}$ satisfies (F3), let us fix $v, w \in E$, and $t \in\left[t_{m}, t_{m+1}\right]$ such that $F$ satisfies inequality (3). Then, according to (6), we have

$$
\begin{aligned}
\left\|F_{m}(t, v, w)\right\| & \leq \alpha(t)\left(1+\|v\|+\left\|w+\sum_{i=0}^{m-1} \int_{t_{i}}^{t_{i+1}} k(t, \tau) y_{i}(\tau) d \tau\right\|\right) \\
& \leq \alpha(t)(1+\|v\|+\|w\|)+\alpha(t) \sum_{i=0}^{m-1} \int_{t_{i}}^{t_{i+1}}\left\|k(t, \tau) y_{i}(\tau)\right\| d \tau .
\end{aligned}
$$

Recalling that $k$ is a positive continuous function (see $(\mathrm{k}))$, for every $i=0, \ldots, m-1$ there exist

$$
k_{m, i}:=\max _{(t, \tau) \in\left[t_{m}, t_{m+1}\right] \times\left[t_{i}, t_{i+1}\right]} k(t, \tau) .
$$


Hence, we get

$$
\begin{aligned}
\left\|F_{m}(t, v, w)\right\| & \leq \alpha(t)(1+\|v\|+\|w\|)+\underbrace{\alpha(t) \sum_{i=0}^{m-1} \int_{t_{i}}^{t_{i+1}} k_{m, i}\left\|y_{i}\right\|_{L^{1}\left(\left[t_{i}, t_{i+1}\right], E\right)}}_{:=\beta_{m}(t)} \\
& \leq \alpha(t)(1+\|v\|+\|w\|)+\beta_{m}(t)(1+\|v\|+\|w\|) \\
& =\alpha_{m}(t)(1+\|v\|+\|w\|),
\end{aligned}
$$

being $\alpha_{m}:=\alpha+\beta_{m} \in L_{+}^{1}\left(\left[t_{m}, t_{m+1}\right]\right)$.

Finally, concerning (F4), let us fix two bounded sets $\Omega_{1}, \Omega_{2} \subset E$, and $t \in\left[t_{m}, t_{m+1}\right]$ such that $F$ satisfies inequality (4). By (6) and the properties of algebraic sub-additivity and nonsingularity of the Hausdorff measure of noncompactness, we have

$$
\begin{aligned}
\chi\left(F_{m}\left(t, \Omega_{1}, \Omega_{2}\right)\right) & \leq h(t)\left[\chi\left(\Omega_{1}\right)+\chi\left(\Omega_{2}+\sum_{i=0}^{m-1} \int_{t_{i}}^{t_{i+1}} k(t, \tau) y_{i}(\tau) d \tau\right)\right] \\
& \leq h(t)\left[\chi\left(\Omega_{1}\right)+\chi\left(\Omega_{2}\right)+\sum_{i=0}^{m-1} \chi\left(\left\{\int_{t_{i}}^{t_{i+1}} k(t, \tau) y_{i}(\tau) d \tau\right\}\right)\right] \\
& =h(t)\left[\chi\left(\Omega_{1}\right)+\chi\left(\Omega_{2}\right)\right],
\end{aligned}
$$

showing the property.

To obtain the existence of mild solutions to our impulsive Cauchy problem $(P)$, we consider an ordered iterative sequence of non-impulsive Cauchy problems, whose mild solutions generate the solutions of the impulsive problem by means of an extension-withmemory process.

Theorem 1. Let $E$ be a real Banach space, $\mathbf{v} \in E,\left\{t_{m}\right\}_{m \in \mathbb{N}}$ with $0 \leq t_{0}<t_{1}<t_{2}<\ldots$ and $\lim _{m \rightarrow+\infty} t_{m}=+\infty$, and $I_{m}: E \rightarrow E$ for $m \in \mathbb{N}^{+}$be given. Suppose that $\mathcal{A}, F$ and $k$, respectively, satisfy hypotheses $(A),(F)$, and $(k)$. Then, problem $(P)$ has at least one mild solution on $\left[t_{0},+\infty[\right.$.

Proof. In association to problem $(P)$, let us consider an iterative sequence of Cauchy problems related to the intervals given by the increasing sequence $\left\{t_{m}\right\}_{m \in \mathbb{N}}$ as follows.

If $m=0$, we consider the problem

$$
\left(P_{0}\right)\left\{\begin{array}{l}
y^{\prime}(t) \in A(t) y(t)+F\left(t, y(t), \int_{t_{0}}^{t} k(t, s) y(s) d s\right), t \in\left[t_{0}, t_{1}\right] \\
y\left(t_{0}\right)=\mathbf{v}
\end{array}\right.
$$

If $m>0$, we define a multimap $F_{m}:\left[t_{m}, t_{m+1}\right] \times E \times E \multimap E$ as

$$
F_{m}(t, v, w):=F_{m-1}\left(t, v, w+\int_{t_{m-1}}^{t_{m}} k(t, \tau) \bar{y}_{m-1}(\tau) d \tau\right), t \in\left[t_{m}, t_{m+1}\right], v, w \in E
$$

(of course, here we mean $F_{0}=F$ ) and a vector in $E$ as

$$
\mathbf{v}_{m}:=\bar{y}_{m-1}\left(t_{m}\right)+I_{m}\left(\bar{y}_{m-1}\left(t_{m}\right)\right),
$$

and consider the problem

$$
\left(P_{m}\right)\left\{\begin{array}{l}
y^{\prime}(t) \in A(t) y(t)+F_{m}\left(t, y(t), \int_{t_{m}}^{t} k(t, s) y(s) d s\right), t \in\left[t_{m}, t_{m+1}\right], \\
y\left(t_{m}\right)=\mathbf{v}_{m},
\end{array}\right.
$$

where $\bar{y}_{m-1} \in C\left(\left[t_{m-1}, t_{m}\right], E\right)$ is a mild solution of problem $\left(P_{m-1}\right)$.

We prove that these mild solutions really exist, by extension. 
First, we consider $m=0$. It is easy to check that, when restricted to $\left[t_{0}, t_{1}\right]$, the hypotheses on the family $\mathcal{A}$ and on the maps $F$ and $k$ come down to the hypotheses of Theorem 5.1 in [34]; actually, that theorem acts on an interval $[0, b]$, but it still holds in $\left[t_{0}, t_{1}\right] \subset\left[0, t_{1}\right]$ (recall that $t_{0}$ is fixed greater or equal to 0 ). Thus we can claim that $\left(P_{0}\right)$ has at least one mild solution $\bar{y}_{0} \in C\left(\left[t_{0}, t_{1}\right], E\right)$, i.e., a continuous function on the interval $\left[t_{0}, t_{1}\right]$ such that

$$
\begin{aligned}
& \bar{y}_{0}(t)=T\left(t, t_{0}\right) \mathbf{v}+\int_{t_{0}}^{t} T(t, s) f_{0}(s) d s, \text { for every } t \in\left[t_{0}, t_{1}\right], \\
& f_{0} \in L^{1}\left(\left[t_{0}, t_{1}\right], E\right), f_{0}(s) \in F\left(s, \bar{y}_{0}(s), \int_{t_{0}}^{s} k(s, \tau) \bar{y}_{0}(\tau) d \tau\right), \text { for a.a. } s \in\left[t_{0}, t_{1}\right] .
\end{aligned}
$$

Let us now fix $m>0$. It is clear that in the interval $\left[t_{m}, t_{m+1}\right]$ we have for $\mathcal{A}$ and $k$ the same situation as in $\left[t_{0}, t_{1}\right]$. Concerning $F_{m}$, notice that it can be rewritten as

$$
F_{m}(t, v, w)=F\left(t, v, w+\sum_{i=0}^{m-1} \int_{t_{i}}^{t_{i+1}} k(t, \tau) \bar{y}_{i}(\tau) d \tau\right), t \in\left[t_{m}, t_{m+1}\right], v, w \in E .
$$

That is, for $F_{m}$ Equation (6) holds. Hence, by Lemma 1 we can claim that $F_{m}$ satisfies (F). Therefore, we can use [34] [Theorem 5.1] again and achieve the existence of a mild solution $\bar{y}_{m}$ to $\left(P_{m}\right)$, i.e., a continuous function on the interval $\left[t_{m}, t_{m+1}\right]$ having the following representation:

$$
\begin{aligned}
& \bar{y}_{m}(t)=T\left(t, t_{m}\right) \mathbf{v}_{m}+\int_{t_{m}}^{t} T(t, s) f_{m}(s) d s, t \in\left[t_{m}, t_{m+1}\right], \\
& f_{m} \in L^{1}\left(\left[t_{m}, t_{m+1}\right], E\right), \\
& f_{m}(s) \in F_{m}\left(s, \bar{y}_{m}(s), \int_{t_{m}}^{s} k(s, \tau) \bar{y}_{m}(\tau) d \tau\right), \text { a.a. } s \in\left[t_{m}, t_{m+1}\right] .
\end{aligned}
$$

We wish to prove that the function $\bar{y}:\left[t_{0},+\infty[\rightarrow E\right.$ defined by

$$
\bar{y}(t):= \begin{cases}\bar{y}_{0}(t), & t \in\left[t_{0}, t_{1}\right] \\ \bar{y}_{m}(t), & \left.t \in] t_{m}, t_{m+1}\right], m>0,\end{cases}
$$

is a mild solution to $(P)$.

To this aim, we firstly put (see (10), (12))

$$
\bar{f}(t):= \begin{cases}f_{0}(t), & t \in\left[t_{0}, t_{1}\right] \\ f_{m}(t), & \left.t \in] t_{m}, t_{m+1}\right], m>0 .\end{cases}
$$

Thus, naturally, $\bar{f} \in L_{l o c}^{1}\left(\left[t_{0},+\infty[, E)\right.\right.$. Further, we show that it is a selector of the multimap $F\left(\cdot, \bar{y}(\cdot), \int_{t_{0}}^{(\cdot)} k(\cdot, \tau) \bar{y}(\tau) d \tau\right)$ almost everywhere in $\left[t_{0},+\infty\right]$. Indeed, note that by (10) we have

$$
\bar{f}_{\mid\left[t_{0}, t_{1}\right]}(s)=f_{0}(s) \in F\left(s, \bar{y}_{0}(s), \int_{t_{0}}^{s} k(s, \tau) \bar{y}_{0}(\tau) d \tau\right), \text { for a.a. } s \in\left[t_{0}, t_{1}\right] .
$$

Thus, by recalling (13) we obtain

$$
\bar{f}(s) \in F\left(s, \bar{y}(s), \int_{t_{0}}^{s} k(s, \tau) \bar{y}(\tau) d \tau\right), \text { for a.a. } s \in\left[t_{0}, t_{1}\right] .
$$


Let us now consider any $m>0$. By (12), we get

$$
\left.\left.\bar{f}_{\left.[] t_{m}, t_{m+1}\right]}(s)=f_{m}(s) \in F_{m}\left(s, \bar{y}_{m}(s), \int_{t_{m}}^{s} k(s, \tau) \bar{y}_{m}(\tau) d \tau\right), \text { for a.a. } s \in\right] t_{m}, t_{m+1}\right] .
$$

By using the expression of $F_{m}$ (see (7)) and the definition of $\bar{y}$ (see (13)), we can therefore write

$$
\begin{gathered}
\bar{f}(s) \in F\left(s, \bar{y}_{m}(s), \int_{t_{m}}^{s} k(s, \tau) \bar{y}_{m}(\tau) d \tau+\sum_{i=0}^{m-1} \int_{t_{i}}^{t_{i+1}} k(s, \tau) \bar{y}_{i}(\tau) d \tau\right) \\
\left.\left.=F\left(s, \bar{y}(s), \int_{t_{0}}^{s} k(s, \tau) \bar{y}(\tau) d \tau\right), \text { for a.a. } s \in\right] t_{m}, t_{m+1}\right] .
\end{gathered}
$$

Now, we can prove that $\bar{y}$ satisfies condition (5) (cf. Definition 1).

If $t \in\left[t_{0}, t_{1}\right]$, then by (13), (9), (14) we have

$$
\bar{y}(t)=T\left(t, t_{0}\right) \mathbf{v}+\int_{t_{0}}^{t} T(t, s) f_{0}(s) d s=T\left(t, t_{0}\right) \mathbf{v}+\int_{t_{0}}^{t} T(t, s) \bar{f}(s) d s .
$$

If $\left.t \in] t_{1}, t_{2}\right]$, then by (13), (11), (8), we get

$$
\begin{aligned}
\bar{y}(t) & =T\left(t, t_{1}\right) \mathbf{v}_{1}+\int_{t_{1}}^{t} T(t, s) f_{1}(s) d s \\
& =T\left(t, t_{1}\right)\left[\bar{y}_{0}\left(t_{1}\right)+I_{1}\left(\bar{y}_{0}\left(t_{1}\right)\right)\right]+\int_{t_{1}}^{t} T(t, s) f_{1}(s) d s .
\end{aligned}
$$

By (9), (T1), (14), (13) we obtain

$$
\begin{aligned}
\bar{y}(t) & =T\left(t, t_{1}\right)\left[T\left(t_{1}, t_{0}\right) \mathbf{v}+\int_{t_{0}}^{t_{1}} T\left(t_{1}, s\right) f_{0}(s) d s\right]+T\left(t, t_{1}\right) I_{1}\left(\bar{y}_{0}\left(t_{1}\right)\right)+\int_{t_{1}}^{t} T(t, s) f_{1}(s) d s \\
& =T\left(t, t_{0}\right) \mathbf{v}+\int_{t_{0}}^{t_{1}} T(t, s) f_{0}(s) d s+T\left(t, t_{1}\right) I_{1}\left(\bar{y}_{0}\left(t_{1}\right)\right)+\int_{t_{1}}^{t} T(t, s) f_{1}(s) d s \\
& =T\left(t, t_{0}\right) \mathbf{v}+T\left(t, t_{1}\right) I_{1}\left(\bar{y}\left(t_{1}\right)\right)+\int_{t_{0}}^{t} T(t, s) \bar{f}(s) d s .
\end{aligned}
$$

Thus, by the same arguments, we can say that if $\left.t \in] t_{m}, t_{m+1}\right]$ for any $m>0$ it holds that

$$
\begin{aligned}
\bar{y}(t) & =T\left(t, t_{m}\right) \mathbf{v}_{m}+\int_{t_{m}}^{t} T(t, s) f_{m}(s) d s \\
& =T\left(t, t_{0}\right) \mathbf{v}+\sum_{i=1}^{m} T\left(t, t_{i}\right) I_{i}\left(\bar{y}\left(t_{i}\right)\right)+\int_{t_{0}}^{t} T(t, s) \bar{f}(s) d s
\end{aligned}
$$

and this concludes the proof.

From careful reading of the proof, it appears that no hypotheses are needed on the impulse functions. Hence, they can be chosen arbitrarily. Thus, if we pick $I_{m}(v)=0$ for every $v \in E$ and $m \in \mathbb{N}^{+}$, we immediately have the following existence result.

Corollary 1. Let $E$ be a real Banach space and $\mathbf{v} \in E$ be given. Suppose that $\mathcal{A}, F$, and $k$, respectively satisfy hypotheses $(A),(F)$, and $(k)$. Then, there exists at least one mild solution on $\left[t_{0},+\infty\right]$ to the Cauchy problem

$$
\left\{\begin{array}{l}
y^{\prime}(t) \in A(t) y(t)+F\left(t, y(t), \int_{t_{0}}^{t} k(t, s) y(s) d s\right), t \geq t_{0} \\
y\left(t_{0}\right)=\mathbf{v}
\end{array}\right.
$$


Of course, in this case, a mild solution is a function $y \in C\left(\left[t_{0},+\infty[, E)\right.\right.$ such that

$$
y(t)=T\left(t, t_{0}\right) \mathbf{v}+\int_{t_{0}}^{t} T(t, s) f(s) d s, t \geq t_{0},
$$

with $f:\left[t_{0},+\infty\left[\rightarrow E\right.\right.$ a $L_{l o c}^{1}$-function on $\left[t_{0},+\infty\left[\right.\right.$ and $f(s) \in F\left(s, y(s), \int_{t_{0}}^{s} k(s, \tau) y(\tau) d \tau\right)$ for a.e. $s \geq t_{0}$.

\section{Example of Application: The Controllability of a Population Dynamics Process with Distributed Delay and Impulses}

In this section, we apply Theorem 1 to the study of the following process with feedback controls described by a parametric integro-differential equation with distributed delay and subject to impulses.

Fixed $0 \leq t_{0}<t_{1}<t_{2}<\ldots$, with $\lim _{m \rightarrow+\infty} t_{m}=+\infty$, and $u_{0} \in L^{2}([0,1])$, we consider the system

$$
(F C P)\left\{\begin{array}{l}
\frac{\partial u}{\partial t}(t, x)=-b(t, x) u(t, x)+g\left(t, u(t, x), \int_{t_{0}}^{t} \frac{e^{-(t-s) / T}}{T} u(s, x) d s\right)+\omega(t, x), \\
\omega(t, \cdot) \in W(u(t, \cdot)), t \geq t_{0}, \quad t \geq t_{0}, t \neq t_{m}, m \in \mathbb{N}^{+}, \text {a.e. } x \in[0,1], \\
u\left(t_{0}, x\right)=u_{0}(x), \text { a.e. } x \in[0,1], \\
u\left(t_{m}^{+}, x\right)=u\left(t_{m}, x\right)+\mathcal{I}_{m}\left(u\left(t_{m}, x\right)\right), m \in \mathbb{N}^{+}, \text {a.e. } x \in[0,1] .
\end{array}\right.
$$

In this model, the real value $u(t, x)$ represents the density of a population depending on time $t$ and place $x$, considering the spatial range normalized to interval $[0,1]$, while the nonlinearity $g:\left[t_{0},+\infty[\times \mathbb{R} \times \mathbb{R} \rightarrow \mathbb{R}\right.$ represents the population development law.

The dependence from the past state of the system is provided by the Volterra integral $\int_{t_{0}}^{t} \frac{e^{-(t-s) / T}}{T} u(s, x) d s$. Indeed, the positive number $T$ gives the width of the action of the kernel, here given by the exponential distribution of probability $\mathcal{K}(\tau)=\frac{e^{-\tau / T}}{T}$. The larger $T$ is, the more the system's memory is extended to past events affecting its present state. Hence, $T$ establishes the width of the range of significance of the delay. Notice that, being the above problem set on the whole half-line, the value of $T$ can be chosen arbitrarily large. This means that the relevance of the delay on the status of the solution trajectory can be chosen arbitrarily, leading to a particularly versatile model.

Moreover, the multimap $W: L^{2}([0,1]) \multimap L^{2}([0,1])$ gives the sets of controls, and the impulse functions $\mathcal{I}_{m}: \mathbb{R} \rightarrow \mathbb{R}$ and $m \in \mathbb{N}^{+}$represent instantaneous external forces acting on the system.

Finally, the death rate and displacement of the population is given by the removal coefficient $-b(t, x)$.

We assume that the function $b:\left[0,+\infty\left[\times[0,1] \rightarrow \mathbb{R}^{+}\right.\right.$satisfies the following conditions:

(b1) $b$ is measurable;

(b2) there exists $s \in L_{l o c}^{1}([0,+\infty[)$ such that

$$
0<b(t, x) \leq s(t)
$$

for every $t \geq 0$, a.e. $x \in[0,1]$; and

(b3) for every $x \in[0,1]$, the function $b(\cdot, x):\left[0,+\infty\left[\rightarrow \mathbb{R}^{+}\right.\right.$is continuous.

Consider the family of linear functions $A(t): L^{2}([0,1]) \rightarrow L^{2}([0,1]), t \geq 0$, defined by

$$
A(t) v(x)=-b(t, x) v(x), v \in L^{2}([0,1]), x \in[0,1]
$$


By [35] [Section 3.1] and [4] [Proposition 3.2 and Remark 3.1], we know that properties (b1)-(b3) imply that the family $\{A(t)\}_{t \geq 0}$ defined by (15) generates the noncompact evolution system

$$
[T(t, s) v](x)=e^{\int_{s}^{t}-b(\sigma, x) d \sigma} v(x), t \geq s \geq 0, v \in L^{2}([0,1]), x \in[0,1] .
$$

Hence, the next proposition holds.

Proposition 1. Under assumptions (b.1)-(b.3), the family $\{A(t)\}_{t \geq 0}$ defined by (15) satisfies property $(A)$.

On the other functions appearing in the model, we assume the next hypotheses.

The function $g:\left[t_{0},+\infty[\times \mathbb{R} \times \mathbb{R} \rightarrow \mathbb{R}\right.$ is such that

(g0) $g(t, v(\cdot), w(\cdot)) \in L^{2}([0,1])$, for every $t \geq t_{0}, v, w \in L^{2}([0,1])$;

(g1) for every $p, q \in \mathbb{R}$, the function $g(\cdot, p, q)$ is (strongly) measurable;

(g2) for a.e. $t \geq t_{0}$, the function $g(t, \cdot, \cdot)$ is continuous;

(g3) there exists a nonnegative function $\varphi \in L_{l o c}^{1}\left(\left[t_{0},+\infty[)\right.\right.$ such that

$$
|g(t, p, q)| \leq \varphi(t),
$$

for a.e. $t \geq t_{0}$ and every $p, q \in \mathbb{R}$; and

(g4) there exists a nonnegative function $m \in L_{l o c}^{1}\left(\left[t_{0},+\infty[)\right.\right.$ such that

$$
\chi_{L^{2}}\left(g\left(t, \Omega_{1}(\cdot), \Omega_{2}(\cdot)\right)\right) \leq m(t)\left[\chi_{L^{2}}\left(\Omega_{1}\right)+\chi_{L^{2}}\left(\Omega_{2}\right)\right] ，
$$

for a.e. $t \geq t_{0}$ and every bounded $\Omega_{1}, \Omega_{2} \subset L^{2}([0,1])$.

The multimap $W: L^{2}([0,1]) \multimap L^{2}([0,1])$ satisfies the properties

(W0) $W$ takes compact convex values;

(W1) $W$ is upper semicontinuous;

(W2) there exists $R>0$ such that, for every $v \in L^{2}([0,1])$,

$$
\|W(v)\|_{L^{2}} \leq R\left(1+\|v\|_{L^{2}}\right),
$$

where $\|W(v)\|_{L^{2}}:=\sup \left\{\|\eta\|_{L^{2}}: \eta \in W(v)\right\}$; and

(W3) there exists $Q>0$ such that

$$
\chi_{L^{2}}(W(\Omega)) \leq Q \chi_{L^{2}}(\Omega),
$$

for every bounded $\Omega \subset L^{2}([0,1])$.

Now, we put:

$$
\begin{aligned}
& v, w:\left[t_{0},+\infty\left[\rightarrow L^{2}([0,1])\right. \text { as }\right. \\
& \qquad v(t)(x)=u(t, x) \text { and } w(t)(x)=\omega(t, x), t \geq t_{0}, x \in[0,1] ; \\
& f:\left[t_{0},+\infty\left[\times L^{2}([0,1]) \times L^{2}([0,1]) \rightarrow L^{2}([0,1])\right. \text { as }\right. \\
& f(t, v, w)(x)=g(t, v(x), w(x)), t \geq t_{0}, v, w \in L^{2}([0,1]), x \in[0,1] ; \\
& I_{m}: L^{2}([0,1]) \rightarrow L^{2}([0,1]), m \in \mathbb{N}^{+} \text {, as } \\
& \qquad I_{m}(v)(x)=\mathcal{I}_{m}(v(x)), v \in L^{2}([0,1]), x \in[0,1]
\end{aligned}
$$


It is easy to check that by these positions we can write the feedback control problem $(F C P)$ as the impulsive Cauchy problem with feedback controls driven by a semilinear integro-differential equation in $L^{2}([0,1])$.

$$
\left\{\begin{array}{l}
v^{\prime}(t)=A(t) v(t)+f\left(t, v(t), \int_{t_{0}}^{t} \frac{e^{-(t-s) / T}}{T} v(s) d s\right)+w(t), t \geq t_{0}, t \neq t_{m}, m \in \mathbb{N}^{+}, \\
w(t) \in W(v(t)), t \geq t_{0}, \\
v\left(t_{0}\right)=v_{0}, \\
v\left(t_{m}^{+}\right)=v\left(t_{m}\right)+I_{m}\left(v\left(t_{m}\right)\right), m \in \mathbb{N}^{+} .
\end{array}\right.
$$

Finally, let us put:

$$
\begin{gathered}
k: \Delta_{\infty} \rightarrow \mathbb{R}^{+} \text {as } \\
k(t, s)=\frac{e^{-(t-s) / T}}{T}, t \geq s \geq t_{0} ; \\
F:\left[t_{0},+\infty\left[\times L^{2}([0,1]) \times L^{2}([0,1]) \multimap L^{2}([0,1])\right. \text { as }\right. \\
F(t, v, w)=f(t, v, w)+W(v), t \geq t_{0}, v, w \in L^{2}([0,1]) .
\end{gathered}
$$

Then, (FCP) can be further rewritten as

$$
\left\{\begin{array}{l}
v^{\prime}(t) \in A(t) v(t)+F\left(t, v(t), \int_{t_{0}}^{t} k(t, s) v(s) d s\right), t \geq t_{0}, t \neq t_{m}, m \in \mathbb{N}^{+}, \\
v\left(t_{0}\right)=v_{0} \\
v\left(t_{m}^{+}\right)=v\left(t_{m}\right)+I_{m}\left(v\left(t_{m}\right)\right), m \in \mathbb{N}^{+},
\end{array}\right.
$$

which is nothing more than a problem of the type $(P)$ in the space $E=L^{2}([0,1])$.

In order to give the controllability of problem $(F C P)$ we need the next result.

Proposition 2. Under assumptions (g0)-(g4) and (W0)-(W3), the multimap F defined in (21) satisfies $(F)$.

Proof. First of all, we observe that by (g0), and recalling (18), one gets $f(t, v, w) \in L^{2}([0,1])$ for every $t \geq t_{0}, v, w \in L^{2}([0,1])$, so that $F$ is well-defined. Moreover, hypothesis (W0) ensures that $F$ satisfies (F0).

With regard to property (F1), it immediately follows from (g1). Indeed, fixed $v, w \in$ $L^{2}([0,1])$, the function $t \mapsto f(t, v, w)=g(t, v(\cdot), w(\cdot))$ is (strongly) measurable. Hence, its translation given by $t \mapsto f(t, v, w)+\omega$, where $\omega$ is an arbitrary element of $W(v)$, is again measurable and represents a measurable selector of $F(\cdot, v, w)$.

Now, fixed $t \geq t_{0}$ for which (g2) and (g3) hold, the map $(v, w) \mapsto f(t, v, w)$ is continuous. In fact, for arbitrarily fixed $\left(v_{0}, w_{0}\right) \in L^{2}([0,1]) \times L^{2}([0,1])$, consider a sequence $\left(v_{n}, w_{n}\right) \rightarrow\left(v_{0}, w_{0}\right)$ in $L^{2}([0,1]) \times L^{2}([0,1])$. Then, by $(\mathrm{g} 2)$ we can write

$$
\left|g\left(t, v_{n}(x), w_{n}(x)\right)-g\left(t, v_{0}(x), w_{0}(x)\right)\right|^{2} \rightarrow 0 \text {, for a.e. } x \in[0,1] .
$$

By (g3), we obtain the following estimate:

$$
\begin{aligned}
\left|g\left(t, v_{n}(x), w_{n}(x)\right)-g\left(t, v_{0}(x), w_{0}(x)\right)\right|^{2} & \leq\left[\left|g\left(t, v_{n}(x), w_{n}(x)\right)\right|+\left|g\left(t, v_{0}(x), w_{0}(x)\right)\right|\right]^{2} \\
& \leq 4 \varphi^{2}(t), \text { for a.e. } x \in[0,1] .
\end{aligned}
$$


We can therefore apply the Lebesgue dominated convergence theorem obtaining

$$
\int_{0}^{1}\left|g\left(t, v_{n}(x), w_{n}(x)\right)-g\left(t, v_{0}(x), w_{0}(x)\right)\right|^{2} d x \rightarrow 0,
$$

and hence

$$
\left\|f\left(t, v_{n}, w_{n}\right)-f\left(t, v_{0}, w_{0}\right)\right\|_{L^{2}}=\sqrt{\int_{0}^{1}\left|g\left(t, v_{n}(x), w_{n}(x)\right)-g\left(t, v_{0}(x), w_{0}(x)\right)\right|^{2} d x} \rightarrow 0 .
$$

The arbitrariness of $\left(v_{0}, w_{0}\right)$ leads to the continuity of $f(t, \cdot, \cdot)$. As a consequence, the multimap $F(t, \cdot, \cdot)$ is the sum of a continuous single-valued function and an upper semicontinuous multimap (see (21) and (W1)), so it is upper semicontinuous as well, i.e., $F$ satisfies (F2).

Let us prove that $F$ satisfies (F3). To this aim, we fix $t \geq t_{0}$ for which (g3) holds and any $v, w \in L^{2}([0,1])$. We clearly have

$$
\|f(t, v, w)\|_{L^{2}}^{2}=\int_{0}^{1}[g(t, v(x), w(x))]^{2} d x \leq \varphi^{2}(t),
$$

so that

$$
\|f(t, v, w)\|_{L^{2}} \leq \varphi(t)\left(1+\|v\|_{L^{2}}+\|w\|_{L^{2}}\right) .
$$

Therefore, by using also (W2), we get

$$
\begin{aligned}
\|F(t, v, w)\|_{L^{2}} & \leq\|f(t, v, w)\|_{L^{2}}+\|W(v)\|_{L^{2}} \\
& \leq \alpha(t)\left(1+\|v\|_{L^{2}}+\|w\|_{L^{2}}\right),
\end{aligned}
$$

where $\alpha(t):=\varphi(t)+R$. The function $\alpha$ belongs to $L_{l o c}^{1}\left(\left[t_{0},+\infty[)\right.\right.$ since $\varphi$ does.

Finally, let us fix $t \geq t_{0}$ for which (g4) holds and any bounded $\Omega_{1}, \Omega_{2} \subset L^{2}([0,1])$. Then, by (21), (18), and (W3), we have

$$
\begin{aligned}
\chi_{L^{2}}\left(F\left(t, \Omega_{1}, \Omega_{2}\right)\right) & \leq \chi_{L^{2}}\left(g\left(t, \Omega_{1}(\cdot), \Omega_{2}(\cdot)\right)\right)+\chi_{L^{2}}\left(W\left(\Omega_{1}\right)\right) \\
& \leq m(t)\left[\chi_{L^{2}}\left(\Omega_{1}\right)+\chi_{L^{2}}\left(\Omega_{2}\right)\right]+Q \chi_{L^{2}}\left(\Omega_{1}\right) \\
& \leq h(t)\left[\chi_{L^{2}}\left(\Omega_{1}\right)+\chi_{L^{2}}\left(\Omega_{2}\right)\right]
\end{aligned}
$$

where $h(t):=m(t)+Q$. As above, $m \in L_{l o c}^{1}\left(\left[t_{0},+\infty[)\right.\right.$ implies $h \in L_{l o c}^{1}\left(\left[t_{0},+\infty[)\right.\right.$, so that $F$ satisfies (F4).

We can now state the main result of this section. In this regard, we should bear in mind that problem (FCP) is controllable if there exists at least one admissible pair for (FCP), that is, a couple $(u, \omega)$ of functions $u, \omega:\left[t_{0},+\infty\left[\times[0,1] \rightarrow \mathbb{R}\right.\right.$ such that: $u(t, \cdot) \in L^{2}([0,1])$ for every $t \geq t_{0} ; u(\cdot, x) \in \mathcal{P C}\left(\left[t_{0},+\infty[, \mathbb{R})\right.\right.$, for all $x \in[0,1] ; u$ satisfies the identity

$$
\begin{aligned}
u(t, x)= & e^{\int_{t_{0}}^{t}-b(\sigma, x) d \sigma} u_{0}(x)+\sum_{t_{0}<t_{m}<t} e^{\int_{t_{m}}^{t}-b(\sigma, x) d \sigma} \mathcal{I}_{m}\left(u\left(t_{m}, x\right)\right)+ \\
& +\int_{t_{0}}^{t} e^{\int_{s}^{t}-b(\sigma, x) d \sigma}\left[g\left(s, u(s, x), \int_{t_{0}}^{s} \frac{e^{-(s-\tau) / T}}{T} u(\tau, x) d \tau\right)+\omega(s, x)\right] d s,
\end{aligned}
$$

for every $t \in\left[t_{0},+\infty\left[, x \in[0,1]\right.\right.$, where $\omega(s, \cdot) \in W(u(s, \cdot))$, a.e. $s \in\left[t_{0},+\infty[\right.$.

Theorem 2. Under assumptions (b1)-(b3), (gO)-(g4), and (W0)-(W3), the problem (FCP) is controllable.

Proof. The hypotheses of the theorem ensure that we can use Proposition 1 and then deduce that the family of linear operators $\{A(t)\}_{t \geq 0}$ defined in (15) satisfies property (A). 
On the other hand, we can also use Proposition 2 and thus infer that the multimap $F$ defined in (21) satisfies property (F). Therefore, we can apply Theorem 1 to problem (22) and obtain the existence of a function $v \in \mathcal{P C}\left(\left[t_{0},+\infty\left[, L^{2}([0,1])\right)\right.\right.$ such that

$$
v(t)=T\left(t, t_{0}\right) v_{0}+\sum_{t_{0}<t_{m}<t} T\left(t, t_{m}\right) I_{m}\left(v\left(t_{m}\right)\right)+\int_{t_{0}}^{t} T(t, s) f(s) d s, t \geq t_{0},
$$

where $f:\left[t_{0},+\infty\left[\rightarrow L^{2}([0,1])\right.\right.$ is a locally summable selector of $F\left(\cdot, v(\cdot), \int_{t_{0}}^{(\cdot)} k(\cdot, \tau) v(\tau) d \tau\right)$ on $\left[t_{0},+\infty[\right.$.

Recalling now conditions (16)-(21), we can rewrite (23) as

$$
\begin{aligned}
u(t, x)= & e^{\int_{t_{0}}^{t}-b(\sigma, x) d \sigma} u_{0}(x)+\sum_{t_{0}<t_{m}<t} e^{\int_{t_{m}}^{t}-b(\sigma, x) d \sigma} \mathcal{I}_{m}\left(u\left(t_{m}, x\right)\right) \\
& +\int_{t_{0}}^{t} e^{\int_{s}^{t}-b(\sigma, x) d \sigma} f(s)(x) d s, t \geq t_{0}, x \in[0,1]
\end{aligned}
$$

where $f(s)=g\left(s, u(s, x), \int_{t_{0}}^{s} \frac{e^{-(s-\tau) / T}}{T} u(\tau, x) d \tau\right)+\omega(s, x)$ and $\omega(s, \cdot) \in W(u(s, \cdot))$, for $x \in[0,1]$ and a.e. $s \geq t_{0}$, concluding the proof.

We conclude the section providing an example of nonlinearity $g$ which satisfies properties $(\mathrm{g} 0)-(\mathrm{g} 4)$.

Example 1. Let us consider the function $g:\left[t_{0},+\infty[\times \mathbb{R} \times \mathbb{R} \rightarrow \mathbb{R}\right.$ defined by

$$
g(t, p, q)=\frac{t}{1+|q|}, t \geq 0, p, q \in \mathbb{R} .
$$

It is easy to check that $(g 0)-(g 3)$ are satisfied by $g$. As for $(g 4)$, we recall that in the space $L^{2}([0,1])$ the Hausdorff measure of noncompactness is equivalent to the measure of noncompactness

$$
\chi_{L^{2}}^{*}(\Omega)=\limsup _{h \rightarrow 0} \sqrt{\int_{0 \in \Omega}^{1}[\theta(x+h)-\theta(x)]^{2} d x}
$$

for every bounded $\Omega \subset L^{2}([0,1])$, according to the relation

$$
\chi_{L^{2}}(\Omega) \leq \chi_{L^{2}}^{*}(\Omega) \leq 2 \chi_{L^{2}}(\Omega)
$$

(see, e.g., [36]).

Then, let us fix $t \geq t_{0}$ and $\Omega_{1}, \Omega_{2}$ bounded subsets of $L^{2}([0,1])$. We have that

$$
\begin{gathered}
\sup _{\theta \in g\left(t, \Omega_{1}, \Omega_{2}\right)} \sqrt{\int_{0}^{1}[\theta(x+h)-\theta(x)]^{2} d x}=\sup _{w \in \Omega_{2}} \sqrt{\int_{0}^{1}\left[\frac{t}{1+|w(x+h)|}-\frac{t}{1+|w(x)|}\right]^{2} d x} \\
=t \sup _{w \in \Omega_{2}} \sqrt{\int_{0}^{1}\left[\frac{|w(x)|-|w(x+h)|}{(1+|w(x+h)|)(1+|w(x)|)}\right]^{2}} d x \leq t \sup _{w \in \Omega_{2}} \sqrt{\int_{0}^{1}[|w(x)|-|w(x+h)|]^{2} d x} \\
\quad \leq t \sup _{w \in \Omega_{2}} \sqrt{\int_{0}^{1} 2[w(x)-w(x+h)]^{2} d x}=\sqrt{2} \sup _{w \in \Omega_{2}} \sqrt{\int_{0}^{1}[w(x)-w(x+h)]^{2} d x .}
\end{gathered}
$$


Thus, bearing in mind (25) and (26), we deduce the next estimate

$$
\begin{aligned}
\chi_{L^{2}}\left(g\left(t, \Omega_{1}, \Omega_{2}\right)\right) & \leq \chi_{L^{2}}^{*}\left(g\left(t, \Omega_{1}, \Omega_{2}\right)\right)=\lim _{h \rightarrow 0} \sup _{\theta \in g\left(t, \Omega_{1}, \Omega_{2}\right)} \sqrt{\int_{0}^{1}[\theta(x+h)-\theta(x)]^{2} d x} \\
& \leq \sqrt{2} t \lim _{h \rightarrow 0} \sup _{w \in \Omega_{2}} \sqrt{\int_{0}^{1}[w(x)-w(x+h)]^{2} d x} \\
& =\sqrt{2} t \chi_{L^{2}}^{*}\left(\Omega_{2}\right) \leq 2 \sqrt{2} t \chi_{L^{2}}\left(\Omega_{2}\right) \\
& \leq 2 \sqrt{2} t\left[\chi_{L^{2}}\left(\Omega_{1}\right)+\chi_{L^{2}}\left(\Omega_{2}\right)\right] .
\end{aligned}
$$

Hence, $(g 4)$ is fulfilled by taking $m(t)=2 \sqrt{2} t, t \geq 0$.

Lastly, we observe that Example 1 can be easily generalized to a function

$$
g(t, p, q)=\frac{\psi(t)}{1+|q|}, t \geq 0, p, q \in \mathbb{R}
$$

where $\psi$ is any $L_{l o c}^{1}$-function.

\section{Conclusions}

This study reveals the existence of mild solutions on the half-line to the impulsive problem $(\mathrm{P})$ under upper-Carathèodory assumptions on the multivalued nonlinearity (cf. properties (F1), (F2)). The extension-with-memory technique here adopted allows impulse functions to be used with no hypotheses, unlike other methods used in the literature, for which those functions are supposed to be at least continuous. Moreover, by means of positions (15)-(21), it is possible to deduce the controllability of the system (FCP) (see Section 4), which is a model for a population dynamics process and that can serve for a wide variety of real phenomena involving a distributed delay.

The methods and some of the results obtained in this paper are expected to be used to analyze problem $(\mathrm{P})$ when the nonlinearity presents a lower semicontinuity type property, and to study the topological properties of the solution set on the half-line under upper or lower semicontinuity type assumptions, alongside all the consequences that results in abstract spaces reflect on models of spread of diseases as well as heat transfer or population dynamics.

Funding: This research has been accomplished within the national group GNAMPA of INdAM and the UMI Group T.A.A. "Approximation Theory and Applications". It has been supported by the INdAM-GNAMPA Project 2020 "Metodi topologici per problemi al contorno associati a certe classi di PDE" and the FRB 2019 "Integrazione, approssimazione, analisi nonlineare e loro applicazioni" by the University of Perugia.

Conflicts of Interest: The author declares no conflict of interest.

\section{References}

1. Aouane, A; Djebali, S.; Taoudi, M.A. Mild solutions of a class of semilinear fractional integro-differential equations subjected to noncompact nonlocal initial conditions. Cubo 2020, 22, 361-377. [CrossRef]

2. Bani Issa, M.S.; Hamoud, A.A.; Ghadle, K.P. Numerical solutions of fuzzy integro-differential equations of the second kind. J. Math. Comput. Sci. 2021, 23, 67-74. [CrossRef]

3. Benchohra, M.; Rezoug, N.; Zhou, Y. Semilinear mixed type integro-differential evolution equations via Kuratowski measure of noncompactness. Z. Anal. Anwend. 2019, 38, 143-156. [CrossRef]

4. Cardinali, T.; Rubbioni, P. The controllability of an impulsive integro-differential process with nonlocal feedback controls. Appl. Math. Comput. 2019, 347, 29-39. [CrossRef]

5. Cimen, E.; Yatar, S. Numerical solution of Volterra integro-differential equation with delay. J. Math. Computer Sci. 2020, 20, 255-263. [CrossRef]

6. El-Sayed, A.M.A.; Al-Issa, Sh. M. Existence of integrable solutions for integro-differential inclusions of fractional order; coupled system approach. J. Nonlinear Sci. Appl. 2020, 13, 180-186. [CrossRef] 
7. Jin, K.-P.; Liang, J.; Xiao, T.-J. Global existence of solutions to some semilinear integro-differential evolution equations with sign-varying kernels. Nonauton. Dyn. Syst. 2020, 7, 65-71. [CrossRef]

8. Kim, D.; Jeong, J.-M. Approximate controllability for semilinear integro-differential control equations with quasi-homogeneous properties. J. Chungcheong Math. Soc. 2021, 34, 189-207.

9. Deng, K.; Wu, Y. Global attractivity of delayed and nonlocal diffusive logistic models with variable coefficients. J. Differ. Equ. 2021, 299, 229-255. [CrossRef]

10. Qin, H.; Wu, F.; Zhang, J.; Mu, C. A linearized compact ADI scheme for semilinear parabolic problems with distributed delay. J. Sci. Comput. 2021, 87, 25. [CrossRef]

11. Arino, O.; Hbid, M.L.; Ait Dads, E. (Eds.) Delay differential equations and applications. In Proceedings of the NATO Advanced Study Institute held at the Cadi Ayyad University, Marrakech, Morocco, 9-21 September 2002; NATO Science Series II: Mathematics, Physics and Chemistry, 205; Springer: Dordrecht, The Netherlands, 2006.

12. Travis, C.C.; Webb, G.F. Existence and stability for partial functional differential equations. Trans. Amer. Math. Soc. 1974, 200, 395-418. [CrossRef]

13. Webb, G.F. Autonomous nonlinear functional differential equations and nonlinear semigroups. J. Math. Anal. Appl. 1974, 46, 1-12. [CrossRef]

14. Bellman, R.; Cooke, K.L. Differential-Difference Equations; Academic Press: New York, NY, USA; London, UK, 1963.

15. Gripenberg, G.; Londen, S.-O.; Staffans, O. Volterra Integral and Functional Equations; Encyclopedia of Mathematics and its Applications, 34; Cambridge University Press: Cambridge, UK, 1990.

16. Hale, J.K.; Verduyn Lunel, S.M. Introduction to Functional-Differential Equations; Applied Mathematical Sciences, 99; Springer: New York, NY, USA, 1993.

17. Iannelli, M.; Pugliese, A. An Introduction to Mathematical Population Dynamics. Along the Trail of Volterra and Lotka; Unitext, 79. La Matematica per il 3+2; Springer: Cham, Switzerland, 2014.

18. Cosentino, C.; Bates, D. Feedback Control in Systems Biology; CRC Press Taylor \& Francis Group: Boca Raton, FL, USA; London, UK New York, NY, USA, 2011.

19. Alsarori, N.A.; Ibrahim, A.G.; Ghadle, K.P. Impulsive mild solutions for nonlocal fractional semilinear differential inclusion with delay in Banach spaces. Dyn. Contin. Discrete Impuls. Syst. Ser. A Math. Anal. 2021, 28, 251-268.

20. Cardinali, T.; Rubbioni, P. Hereditary evolution processes under impulsive effects. Mediterr. J. Math. 2021, 18, 1-26. [CrossRef]

21. Cichoń, M.; Satco, B. Existence theory for semilinear evolution inclusions involving measures. Math. Nachr. 2017, 290, 1004-1016. [CrossRef]

22. Di Piazza, L.; Marraffa, V.; Satco, B. Closure properties for integral problems driven by regulated functions via convergence results. J. Math. Anal. Appl. 2018, 466, 690-710. [CrossRef]

23. Kulmanakova, M.M.; Obukhovskii, V.V.; Ulyanova, E.L. On a nonlocal Cauchy problem for a semilinear impulse differential inclusion with a causal operator in a Banach space. Vestn. Voronezh. Gos. Univ. Ser. Fiz. Mat. 2021, 1,92-109.

24. Luo, Y.; Wang, W. Existence for impulsive semilinear functional differential inclusions. Qual. Theory Dyn. Syst. 2021, 20, 1-8. [CrossRef]

25. Rubbioni, P. Asymptotic stability of solutions for some classes of impulsive differential equations with distributed delay. Nonlinear Anal. Real World Appl. 2021, 61, 103324. [CrossRef]

26. Lakshmikantham, V.; Bainov, D.D.; Simeonov, P.S. Theory of Impulsive Differential Equations; Series in Modern Applied Mathematics, 6; World Scientific Publishing Co., Inc.: Teaneck, NJ, USA, 1989.

27. Samoǐlenko, A.M.; Perestyuk, N.A. Impulsive differential equations. In World Scientific Series on Nonlinear Science; Series A: Monographs and Treatises, 14; World Scientific Publishing Co., Inc.: River Edge, NJ, USA, 1995.

28. Cardinali, T.; Rubbioni, P. Impulsive semilinear differential inclusions: topological structure of the solution set and solutions on non-compact domains. Nonlinear Anal. 2008, 69, 73-84. [CrossRef]

29. Benedetti, I.; Rubbioni, P. Existence of solutions on compact and non-compact intervals for semilinear impulsive differential inclusions with delay. Topol. Methods Nonlinear Anal. 2008, 32, 227-245.

30. Pazy, A. Semigroups of Linear Operators and Applications to Partial Differential Equations; Applied Mathematical Sciences, 44; Springer: New York, NY, USA, 1983.

31. Krein, S.G. Linear Differential Equations in Banach Space; Translated from the Russian by J. M. Danskin; Translations of Mathematical Monographs; American Mathematical Society: Providence, RI, USA, 1971; Volume 29.

32. Ayerbe Toledano, J.M.; Domínguez Benavides, T.; López Acedo, G. Measures of Noncompactness in Metric Fixed Point Theory; Operator Theory: Advances and Applications, 99; Birkhäuser Verlag: Basel, Switzerland, 1997.

33. Kamenskii, M.; Obukhovskii, V.; Zecca, P. Condensing Multivalued Maps and Semilinear Differential Inclusions in Banach Spaces; De Gruyter Ser. Nonlinear Anal. Appl. 7; Walter de Gruyter: Berlin, Germany; New York, NY, USA, 2001.

34. Bungardi, S.; Cardinali, T.; Rubbioni, P. Nonlocal semilinear integro-differential inclusions via vectorial measures of noncompactness. Appl. Anal. 2017, 96, 2526-2544. [CrossRef]

35. Malaguti, L.; Rubbioni, P. Nonsmooth feedback controls of nonlocal dispersal models. Nonlinearity 2016, 29, 823-850. [CrossRef]

36. Appell, J. Measures of noncompactness, condensing operators and fixed points: an application-oriented survey. Fixed Point Theory 2005, 6, 157-229. 